OPEN ACCESS

Edited by:

Ziad Daoud,

University of Balamand, Lebanon

Reviewed by:

Bartolome Moya Canellas, University of Florida, United States

Stephen E. Mshana,

Catholic University of Health and Allied Sciences (CUHAS),

Tanzania

${ }^{*}$ Correspondence:

Shiri Navon-Venezia shirinv@ariel.ac.i

these authors have contributed equally to this work

Specialty section: This article was submitted to Antimicrobials, Resistance and Chemotherapy,

a section of the journa

Frontiers in Microbiology

Received: 26 June 2019 Accepted: 15 October 2019

Published: 30 October 2019

Citation:

Shnaiderman-Torban A Steinman A, Meidan G, Paitan Y, Abu Ahmad W and Navon-Venezia S (2019) Petting Zoo Animals as an

Emerging Reservoir of Extended-Spectrum $\beta$-Lactamase and AmpC-Producing

Enterobacteriaceae

Front. Microbiol. 10:2488 doi: 10.3389/fmicb.2019.02488

\section{Petting Zoo Animals as an Emerging Reservoir of Extended-Spectrum $\beta$-Lactamase and AmpC-Producing Enterobacteriaceae}

\author{
Anat Shnaiderman-Torban', Amir Steinman 1†, Gal Meidan', Yossi Paitan ${ }^{2,3}$, \\ Wiessam Abu Ahmad ${ }^{4}$ and Shiri Navon-Venezia ${ }^{5 * t}$ \\ ' Koret School of Veterinary Medicine, The Robert H. Smith Faculty of Agriculture, Food and Environment, The Hebrew \\ University of Jerusalem, Rehovot, Israel, ${ }^{2}$ Department of Clinical Microbiology and Immunology, Sackler Faculty of Medicine, \\ Tel Aviv University, Tel Aviv, Israel, ${ }^{3}$ Clinical Microbiology Lab, Meir Medical Center, Kfar Saba, Israel, ${ }^{4}$ Hebrew \\ University-Hadassah Braun School of Public Health and Community Medicine, Jerusalem, Israel, ${ }^{5}$ Department of Molecular \\ Biology, Faculty of Natural Sciences and Adelson School of Medicine, Ariel University, Ariel, Israel
}

Extended spectrum beta-lactamases and AmpC-producing Enterobacteriaceae (ESBL/AmpC-E) have become a great concern in both human and veterinary medicine. One setting in which this risk could be particularly prominent is petting zoos, in which humans, especially children, directly and indirectly interact with the animals. Yet, while the zoonotic transmission of various Enterobacteriaceae has been reported previously in petting zoos, reports on ESBL/AmpC-E shedding in this setting is currently lacking, despite the high potential risk. To fill this knowledge gap, we conducted a prospective cross-sectional study to explore the prevalence, molecular epidemiology, and risk for shedding of ESBL/AmpC-E in petting zoos. We performed a prospective cross-sectional study in eight petting zoos. Altogether, we collected 381 fecal and bodysurface samples from 228 animals, broth-enriched them, and then plated them onto $\mathrm{CHROMagar} \mathrm{ESBL}$-plates for $\mathrm{ESBL} / \mathrm{AmpC}-\mathrm{E}$ isolation. Next, we identified the isolated species and tested their susceptibility to various antibiotics using the Vitek-2 system, determined bacterial relatedness by multilocus sequence typing (MLST), and identified ESBL/AmpC genes by using PCR and sequencing. Finally, we asked petting zoo owners and veterinarians to complete questionnaires, which we then analyzed to evaluate risk factors for ESBL/AmpC-E shedding. We found that ESBL/AmpC-E shedding is an important, currently oversighted risk in petting zoos, as the overall shedding rate was 12\% (35 isolates, including 29\% ESBL-producers, 34\% AmpC-producers, and $37 \% \mathrm{ESBL}$ and AmpC-producers). The isolated bacteria included Enterobacter cloacae (55\%), Escherichia coli (31\%), and Citrobacter freundii (14\%), with diverse ESBL genes. MLST revealed diverse sequence types (STs), including the highly virulent Enterotoxigenic ST656 and the Uropathogenic ST127 E. coli strains, indicating complex 
epidemiology with inter-animal bacterial transmission. Shedding was associated with petting permission and antibiotic treatment in the petting zoo $(\mathrm{OR}=7.34)$, which were identified as risk factors for ESBL/AmpC shedding. Our findings highlight petting zoos as a source for antibiotic-resistant ESBL/AmpC-producing bacteria, including highly virulent, disease-associated MDR E. coli strains. As this risk has not been previously described in detail, it calls for the implementation of infection control and active surveillance programs in petting zoos and raises the need for a comprehensive guideline to restrain this emerging concern.

Keywords: petting zoos, animals, ESBL, AmpC, environmental shedding, Enterobacteriaceae, risk factors

\section{INTRODUCTION}

Petting zoos - either permanent or temporary - are popular attraction sites that allow both direct and indirect exposure of children and adults to diverse animals (Steinmuller et al., 2006). Despite the educational and entertainment value of such interactions, petting zoos raise a significant concern regarding the zoonotic transmission of pathogens due to contact with animals, mainly through the oral-fecal route (Conrad et al., 2016). Indeed, the US Centers for Disease Control and Prevention (CDC) has published recommendations on "how to stay healthy at animal exhibits"1. Previous reports of public health risks and zoonosis originating from petting zoos, mostly in North America, typically describe either the transmission of highly virulent bacterial pathogens, including Escherichia coli and Salmonella outbreaks in petting zoos (Friedman et al., 1998; Goode et al., 2009), or risk behaviors for disease transmission (Weese et al., 2007; Erdozain et al., 2013).

In the past two decades, the global incidence of plasmidmediated AmpC and extended spectrum beta-lactamases (ESBL)producing Enterobacteriaceae (ESBL/AmpC-E) has increased constantly in both humans and animals (Schwaber et al., 2006; Barco et al., 2015; Leonard et al., 2015). Environmental shedding of ESBL/AmpC-E by farm animals, such as cattle, poultry, and swine has been widely investigated in the past (Horton et al., 2011), but it is alarmingly understudied in petting zoos. In a single study, ESBL-producing $E$. coli were isolated from feces of petting zoo animals (Conrad et al., 2018), but larger studies on the incidence and risk factors for ESBL/AmpC-E shedding in petting zoos are lacking. Due to the direct contact between visitors (mainly children) and animals, identifying and characterizing the presence of these antibiotic-resistant bacteria on the body surface of the animals and the possible environmental shedding is of great public importance. Accordingly, in this prospective study, our aim was to determine the prevalence of ESBL/AmpCE shedding in various petting-zoo animal species, characterize the molecular epidemiology of the isolates, and define the risk factors for shedding.

Addressing the emerging threat of antibiotic-resistant bacteria in petting zoo animals requires a "One Health" perspective and, therefore, the data from this study are crucial to the fight against the spread of resistance.

\footnotetext{
${ }^{1}$ https://www.cdc.gov/features/animalexhibits/index.html
}

\section{MATERIALS AND METHODS}

\section{Petting Zoos and Study Design}

We conducted a prospective cross-sectional study in eight permanent petting zoos across Israel (December 2016-May 2017), chosen randomly. The study was approved by the Internal Ethics Committee of the Koret School of Veterinary Medicine, Israel (Protocol KSVM-VTH/25_2016), and was made possible through a respectable collaboration with the facility owners and veterinarians. We recruited petting zoos based on owners' cooperation, considering the appropriate sample size. In order to examine diverse risk factors for shedding, we chose petting zoos which differed in characteristics (schools, exhibition, in a zoo property and ambulatory). In interviews conducted with the owners, we collected demographic and medical data, throughout owners' questioners. Data included the total number of animals in each facility, the number of animal species, the type of veterinary care, petting and feeding policies by visitors and employees, the number of employees, and the average daily number of visitors. Data on each sampled animal included its class, species, diet, and sex, and antibiotic treatments that had been received during the past year, according to owners' questioner.

\section{Bacteria Sampling, Isolation, Identification, and Antibiotic Susceptibility Testing}

In each petting zoo, we sampled the maximum number of animals from diverse species. The goal was to sample all animals housed in the petting zoo, whereas in practice we sampled the animals which the owners approved to sample, mainly due to safety considerations. Sampling was performed during morningnoon hours. We collected fecal specimens from the close vicinity of the animal and analyzed them only if we could link them, through direct observation, to a specific animal. According to the decision of the owners and the ability to safely approach the animal, we also collected surface specimens from the skin, fur, or feathers by rubbing a sterile cotton swab, pre-moistened with saline, on the surface of the animal, in the back area, for at least $10 \mathrm{~s}$. The sampling area was proportional to the size of each sampled animal.

Samples were stored at room temperature, in the commercial transport gel and were processed within $24 \mathrm{~h}$ of sampling. All samples were inoculated into 2 - $\mathrm{mL}$ of a brain-heart infusion 
enrichment broth, so as to increase the sensitivity of ESBL and AmpC-E detection (Murk et al., 2009). After incubation of 18$24 \mathrm{~h}$ at $37^{\circ} \mathrm{C}$, the enriched cultures were plated $(10 \mu \mathrm{L})$ onto Chromagar ESBL plates (Hy-Labs, Rehovot, Israel). Colonies that appeared after an overnight incubation at $37^{\circ} \mathrm{C}$ were recorded, and one colony of each distinct color was re-streaked onto a fresh Chromagar ESBL plate to obtain a pure culture. Next, the pure $\mathrm{ESBL} / \mathrm{AmpC}-\mathrm{E}$ suspected isolates were stored at $-80^{\circ} \mathrm{C}$ stocks for further workup. All isolates were subjected to Vitek-2 for species identification and antibiotic susceptibility testing (ASTN270 Vitek2 card, BioMérieux, Inc., Marcy-l'Etoile, France). The identification of Enterobacter and Citrobacter species was verified using 16S rRNA gene sequencing and an RDP database comparison (Cole et al., 2005). ESBL and AmpC production were confirmed using combination disc diffusion confirmatory assays and interpreted according to the CLSI guidelines (CLSI, 2017, 27th edition). We adopted the guidelines for E. coli ESBL confirmatory assay by disc diffusion, and implemented it for Enterobacter cloacae and for Citrobacter freundii. All E. cloacae and for C. freundii isolates were automatically defined as AmpC producers, due to intrinsic resistance. Plasmid mediated AmpC production was tested via cefoxitin and resistance to second generation cephalosporin.

\section{Genotyping and Detection of $\beta$-Lactamase Genes of ESBL/AmpC-E}

To determine the genetic relatedness between isolates belonging to the same species, genotyping was performed using an Enterobacterial repetitive intergenic consensus (ERIC) PCR amplification with the following primer: 5'-AAG TAAGTGACTGGGGTGAGCG-3' (Versalovic et al., 1991). Results were analyzed using GelJ software (Heras et al., 2015) and all strains exhibiting a distinct ERIC PCR pattern were subjected to multilocus sequence type (MLST) using schemes for E. coli, E. cloacae, and C. freundii, as described previously (Diancourt et al., 2005; Bai et al., 2012; Miyoshi-Akiyama et al., 2013). Sequences of new gene alleles and sequence types (STs) were submitted to and assigned by PubMLST ${ }^{2}$.

Extended spectrum beta-lactamases and AmpC $\beta$-lactamase genes were identified by PCRs and sequencing. Isolates were examined for the presence of $b l a_{\mathrm{CMY}-1}, b l a_{\mathrm{CMY}-2}$ (Kim et al., 2005), bla ${ }_{\mathrm{CTX}-\mathrm{M}}$ group (Woodford et al., 2006), bla $a_{\mathrm{OXA}-1}$, $b l a_{\mathrm{OXA}-2}, b l a_{\mathrm{OXA}-10}$ (Lin et al., 2012), bla $a_{\mathrm{TEM}}$, and bla $a_{\mathrm{SHV}}$ groups (Tofteland et al., 2007). Genes identified as bla and bla $\mathrm{CTX}-\mathrm{M}-9$ groups were sequenced to identify the specific gene allele, using the following primers (designed in this study): $\quad b l a_{\mathrm{CTX}-\mathrm{M}-1} \mathrm{~F}-$ ATGGTTAAAAAATCACTGCG and $b^{b} a_{\mathrm{CTX}-\mathrm{M}-1} \mathrm{R}$ TTACAAACCGTTGGTGACG, bla $a_{\mathrm{CTX}-\mathrm{M}-9} \mathrm{~F}-$ ATGGTGACAAAGAGAGTGCAAC and bla $a_{\mathrm{CTX}-\mathrm{M}-9} \mathrm{R}$ TTACAGCCCTTCGGCGATGA, respectively.

\section{Statistical and Risk Factor Analyses}

The minimal sample size (number of animals sampled) was calculated using WinPepi, based on an estimated shedding rate of $10 \%$ for ESBL-E in community companion animals in

${ }^{2}$ https://pubmlst.org/
Israel (A. Shnaiderman-Torban, unpublished), with a confidence level of $95 \%$ and an acceptable difference of $5 \%$, resulting in $n=139$. Statistical analyses were performed using the IBM STATISTICS SPSS software (SPSS Version 24; SPSS Inc., Chicago, IL, United States). Data distribution was examined by testing whether the Skewness and Kurtosis equal zero and by performing the Shapiro-Wilk's test. Continuous variables were analyzed using $t$-tests or Mann-Whitney $U$-tests, according to the distribution of the variable. Categorical variables were analyzed using the Fisher's exact test or the Pearson chi-square test, as appropriate. In all statistical analyses, $p \leq 0.05$ indicated significance. A multiple logistic regression model, using the ENTER method, was applied for ESBL/AmpC-E shedding using variables with $p \leq 0.2$.

\section{RESULTS}

\section{Characterization of Petting Zoos and Animal Populations}

The study population included animals in eight petting zoos in Israel, which were diverse in type, size, and other characteristics (Table 1). Overall, 228 animals (42 species) were sampled for ESBL/AmpC gut-shedding, including 161 mammals (71\%, 23 species), 47 reptiles (20\%, 12 species), and 20 avian species (9\%, 7 species). Altogether, 381 specimens were collected from these animals, including fecal samples from 52 animals, surface samples (skin/fur/feathers) from 23 animals, and both fecal and surface samples from 153 animals.

\section{Prevalence of ESBL and/AmpC-Producing Enterobacteriaceae (ESBL/AmpC-E)}

Of the 228 sampled animals, $12 \%(n=28$, CI 95\% $8-17 \%)$ carried at least one strain of ESBL/AmpC-E and 25\% co-carried more than one antibiotic-resistant strain (Figure 1, Table 2, and Supplementary Table S1). Carriage rates within different petting zoos varied significantly, from 0 to $22 \%$ (Table 2). Overall, 35 ESBL/AmpC-E isolates were recovered from 28 animals, of which $77 \%$ were from feces samples and $23 \%$ were from surface samples ( $n=27$ and eight samples, respectively; Figure 1, Table 2, and Supplementary Table S1).

Of the 153 animals that were sampled from both feces and body surface, 15 animals were positive for ESBL/AmpCE only in fecal samples, four animals were positive only in surface samples, and two animals were positive in both fecal and surface samples: a turtle from petting zoo \#8, which carried two different E. cloacae strains on the skin (ST102) and in the feces (ST1152), with different resistance phenotypes (isolates p151.2 and p152.2; Supplementary Table S1); and a meerkat from petting zoo \#6, which carried the same ESBL-producing E. coli strain, ST648, on its fur and in its feces (isolates p381.2 and p382.2; Supplementary Table S1).

Of the 42 animal species that were sampled, 19 species carried ESBL/AmpC-E (11 mammals, 7 reptiles, and one avian species). To the best of our knowledge, for 13 of these animal species, this 
TABLE 1 | Characteristics of the eight petting zoos included in this study.

\begin{tabular}{|c|c|c|c|c|c|c|c|c|}
\hline \multirow[t]{2}{*}{ Petting zoo } & \multirow[t]{2}{*}{$\begin{array}{l}\text { Type of } \\
\text { facility }\end{array}$} & \multirow[t]{2}{*}{$\begin{array}{l}\text { per zoo } \\
\text { per zoo }\end{array}$} & \multirow[t]{2}{*}{$\begin{array}{l}\text { No. of animal } \\
\text { species }\end{array}$} & \multirow[t]{2}{*}{$\begin{array}{c}\text { No. of } \\
\text { employees }\end{array}$} & \multirow[t]{2}{*}{$\begin{array}{l}\text { Average daily } \\
\text { visitors }\end{array}$} & \multicolumn{2}{|c|}{$\begin{array}{l}\text { Permitted } \\
\text { policy }\end{array}$} & \multirow[t]{2}{*}{$\begin{array}{l}\text { No. of animals treated with } \\
\text { antibiotics at sampling }(\%)^{\mathrm{a}}\end{array}$} \\
\hline & & & & & & Petting & Eating & \\
\hline 1 & Zoo property & $<50$ & 15 & 6 & 50 & + & - & 0 \\
\hline 2 & Exhibition ${ }^{b}$ & & 20 & 3 & 50 & - & + & 8/19 (42) \\
\hline 3 & Zoo property & & 10 & 8 & 150 & + & - & 0 \\
\hline 4 & Ambulatoryc & $50-100$ & 30 & 2 & 10 & + & - & $4 / 24(17)$ \\
\hline 5 & School property & & 20 & 3 & 50 & + & + & $2 / 46(4)$ \\
\hline 6 & School property & & 30 & 2 & 10 & + & - & $2 / 53(4)$ \\
\hline 7 & Private & $>100$ & 20 & 9 & 70 & + & + & 0 \\
\hline 8 & Zoo property & & 35 & 14 & $>100$ & + & - & 5/38 (13) \\
\hline
\end{tabular}

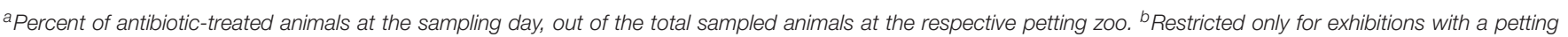
prohibition policy. ' Used for celebrations of children parties and festivals.

is the first report describing ESBL/AmpC-E shedding (Figure 1 and Supplementary Table S1).

More than half $(55 \%, n=19)$ of the $35 \mathrm{ESBL} / \mathrm{AmpC}$-producing isolates belonged to the genera $E$. cloacae complex, while the rest were identified as E. coli $(31 \%, n=11)$ and C. freundii $(14 \%$, $n=5)$. The isolates encompassed strains that produce both ESBLs and $\mathrm{AmpC}(37 \%, n=13)$, AmpC alone (34\%, $n=12)$, or ESBL alone $(29 \%, n=10)$. E. cloacae was the only species that was associated with gut shedding ( $p=0.019$; Figure 1, Table 2, and Supplementary Table S1).

\section{Antibiotic Susceptibility Profiles of ESBL/AmpC-E}

The antibiotic susceptibility profiles of the ESBL/AmpC-E isolates were diverse (Supplementary Table S1). For isolates producing both ESBLs and AmpC $(n=13)$, resistance rates were $100 \%$ to amoxicillin/clavulanate, $38 \%$ to fosfomyicin, and $31 \%$ to nitrofurantoin (intermediate susceptibility). All isolates were susceptible to carbapenems, gentamicin, and trimethoprim/sulfamethoxazole. For AmpC-E $(n=12)$, resistance rates were $100 \%$ to amoxicillin/clavulanate, $33 \%$ to fosfomyicin, $17 \%$ to ofloxacin, $8 \%$ to ciprofloxacin, and $25 \%$ to nitrofurantoin (intermediate susceptibility). All AmpC-E isolates were susceptible to gentamicin and trimethoprim/sulfamethoxazole. For ESBL-E $(n=10)$, resistance rates were $50 \%$ to trimethoprim/sulfamethoxazole, $20 \%$ to ofloxacin, $33 \%$ to ciprofloxacin, and $10 \%$ to gentamicin. All ESBL-E isolates were susceptible to amoxicillin/clavulanate (one isolate had an intermediate susceptibility), piperacillin/tazobactam, fosfomyicin, and nitrofurantoin (Supplementary Table S1).

\section{Genotyping of the ESBL/AmpC-E Isolates and Resistance Genes}

To understand the genetic relatedness between ESBL/AmpC-E strains within and between different petting zoos, we performed ERIC PCR, followed by MLST analysis. We performed ERIC PCR on 30 isolates that were kept and stored successfully. E. cloacae revealed 14 isolates with 13 different clusters, E. coli revealed
11 isolates with six different clusters and Citrobacter freundii revealed five isolates with three different clusters (Supplementary Figure S1). MLST analysis performed on E. cloacae complex demonstrates that isolates belonged to multiple STs, of which seven are known STs, four (ST1151-ST1154) were assigned as new STs possessing new allele combinations, and one (ST1189) was assigned as a new ST possessing five new alleles: dnaA-329, fusA-215, gyrB-356, leuS-402, and rplB-153 (Figure 1).

Escherichia coli was the second most prevalent $\beta$-lactamaseproducing species $(31 \%, n=11 / 35)$, in which the majority of the isolates $(90 \%, n=10 / 11)$ were ESBL-producers and only one strain (ST224) was an AmpC-producer carrying a bla $\mathrm{CMY}-2$. The ESBL genes detected in this species belonged to either the bla $a_{\mathrm{CTX}-\mathrm{M}-1}$ group (three isolates: bla $a_{\mathrm{CTX}-\mathrm{M}-28}$ ), the $b l a_{\mathrm{CTX}-\mathrm{M}-9}$ group (one isolate: $b l a_{\mathrm{CTX}-\mathrm{M}-14}$ ), and the bla $a_{\mathrm{SHV}}$ group (bla $a_{\mathrm{SHV}-12}, b l a_{\mathrm{SHV}-31}, b l a_{\mathrm{SHV}-2}$, and bla $a_{\mathrm{SHV}-2 \mathrm{a}}$, each detected in a different, single isolate). The 11 ESBL-producing E. coli isolates belonged to six known STs, including ST656 (four ring-tailed coatis from petting zoo \#7), ST648 (two meerkats from petting zoo \#6), ST127 (two ferrets from petting zoo \#8), and three single-isolate STs: ST4981, ST2521, and ST224 (Figure 1). Neither of the E. coli isolates belonged to the worldwide ESBLproducing E. coli ST131 lineage.

The third species recovered from animals was the AmpCproducer C. freundii (five isolates; Figure 1 and Supplementary Table S1). Three isolates carried the bla $a_{\mathrm{CMY}-2}$ gene; two of these isolates were also ESBL-producers, one produced bla $a_{\mathrm{CTX}-\mathrm{M}-28}$, and the other ESBL gene was not identified. Genotyping revealed the presence of two different strains shed by different animal species in zoo \#6 (ST124 and ST367), and one C. freundii strain, assigned with a new ST, ST479, encoding two new alleles: aspC-177 and dnaG-167, shed by three individual coatis housed together in petting zoo \#7 (Figure 1), suggesting interanimal spread.

\section{Risk Factor Analysis for ESBL/AmpC-E Shedding}

In a Univariable analysis, the shedding of an ESBL-E or an AmpC-E or ESBL/AmpC-E by an individual animal was 


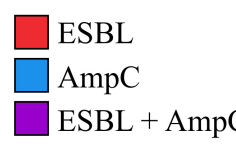

$$
\text { C }
$$$$
\text { * }
$$

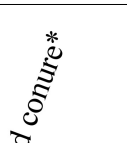

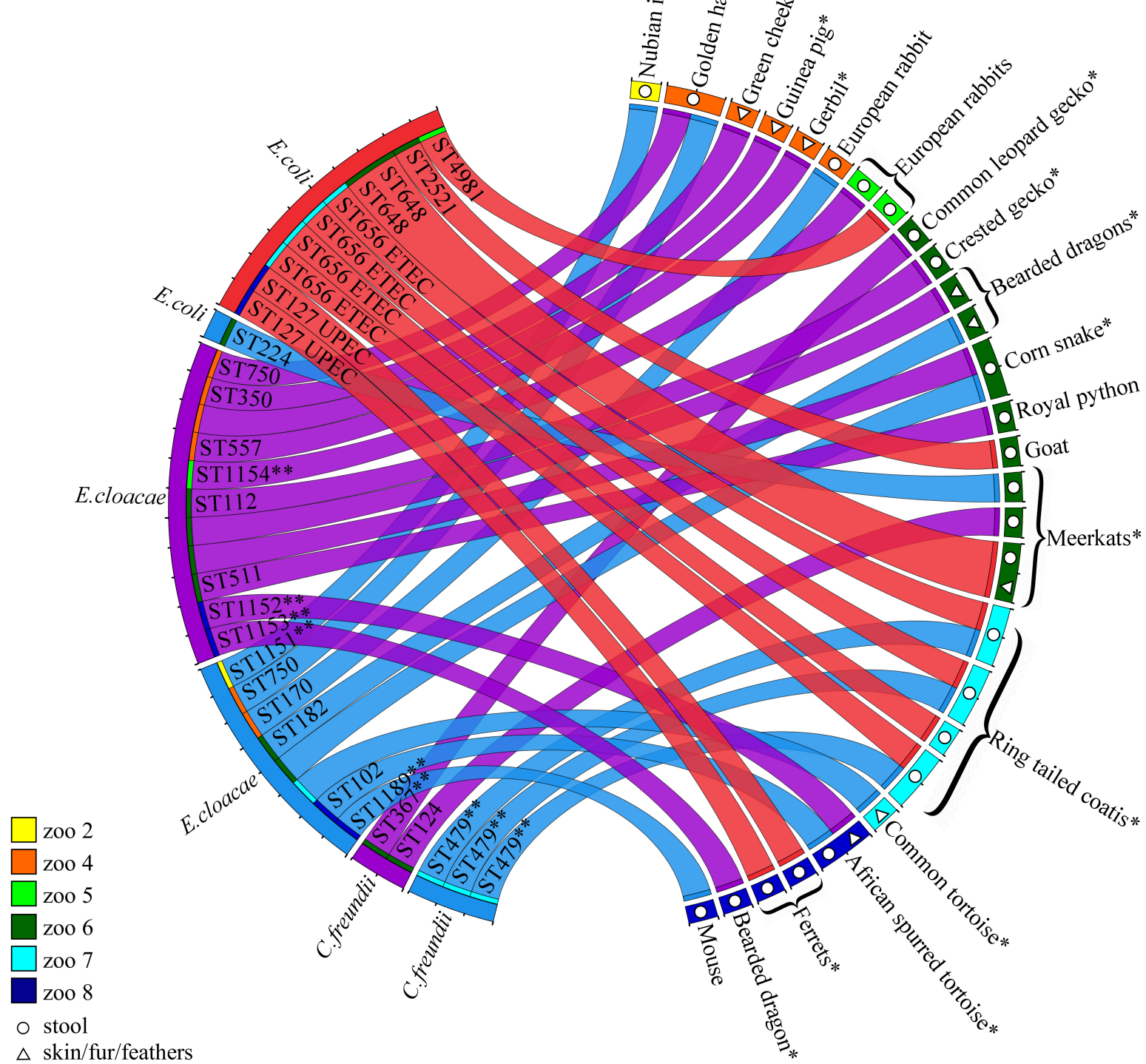

FIGURE 1 | ESBL/AmpC-E isolates recovered from petting zoo animals. Circos diagram presenting the 35 ESBL/AmpC-E isolates (left) recovered from 28 animals and their housing petting zoos (right; each animal is represented by a colored square). The bacterial species, sequence type (ST), resistance phenotype (ESBL, AmpC, or both; represented by the color of the ribbon), and isolation source [feces, represented by open circles, or surface (skin/fur/feathers), represented by open triangles] are designated for each isolate. Animal species described here for the first time as shedding ESBL/AmpC-E are designated with an *. New bacterial STs are designated with **. ESBL/AmpC-E isolates that are represented by a ribbon with an unassigned ST represent bacteria that were not preserved for further investigation due to technical reasons. UPEC, Uropathogenic E. coli; ETEC, Enterotoxigenic E. coli.

found to be significantly associated with antibiotic treatment $(p=0.038, p=0.011$, and $p=0.029$, respectively; Table 3). Overall, $11 \%$ of the sampled animals $(n=25 / 228)$ were treated with antibiotics, including trimethoprim/sulfamethoxazole, cephalosporins, doxycycline, metronidazole, chloramphenicol, and the veterinarian quinolones enrofloxacin and marbofloxacin. ESBL/AmpC-E shedding was not associated with any specific antibiotic agent. AmpC-E and ESBL-E shedding were associated with antibiotic treatment, the permitted petting policy, and the petting zoo sampled (Table 3). These factors were included in a logistic regression model, which revealed that antibiotic therapy is a risk factor for ESBL/AmpC-E shedding (OR $=7.34$, 95\% CI 1.88 28.56). In addition, petting zoo $\# 2$ was found to be a protective factor against ESBL/AmpC-E carriage $(\mathrm{OR}=0.078$, $95 \%$ 0.007-0.92). 
TABLE 2 | Shedding rates of ESBL and AmpC-producing Enterobacteriaceae in petting zoos.

\begin{tabular}{|c|c|c|c|c|c|}
\hline \multirow[t]{2}{*}{ Petting zoo } & \multirow{2}{*}{$\begin{array}{c}\text { No. of } \\
\text { sampled } \\
\text { animals }\end{array}$} & \multicolumn{3}{|c|}{ Number of positive ESBL/AmpC shedding animals (\%) and bacterial species } & \multirow{2}{*}{$\begin{array}{l}\text { Total No. of } \\
\text { ESBL/AmpC } \\
\text { carriers (\%) }\end{array}$} \\
\hline & & Mammals & Reptiles & Avian species & \\
\hline 1 & 10 & $0 / 10(0)$ & Not sampled & Not sampled & 0 \\
\hline 2 & 19 & 1/14 (7) E. cloacae complex (1) & $0 / 2(0)$ & $0 / 3(0)$ & $1 / 19(5)$ \\
\hline 3 & 15 & $0 / 15(0)$ & Not sampled & Not sampled & 0 \\
\hline 4 & 24 & 4/17 (24) $)^{a}$ E. cloacae complex (5) & $0 / 4(0)$ & 1/3 (30) E. cloacae complex (1) & $5 / 24(20)$ \\
\hline 5 & 46 & $\begin{array}{c}\text { 2/33 (6) E. cloacae complex (1); } \\
\text { E. coli }(1)\end{array}$ & $0 / 5(0)$ & $0 / 8(0)$ & $2 / 46(4)$ \\
\hline 6 & 53 & 4/25 (16) ${ }^{\mathrm{a}}$ C. freundii (1); E. coli (4) & $\begin{array}{c}\text { 6/23 (30) })^{\mathrm{a}} \text { E. cloacae complex (6); } \\
\text { C. freundii (1) }\end{array}$ & $0 / 5(0)$ & $10 / 53(19)$ \\
\hline 7 & 23 & 4/16 (25) ${ }^{\text {b }}$ C. freundii (3); E. coli (4) & 1/6 (17) E. cloacae complex (1) & $0 / 1(0)$ & $5 / 23(22)$ \\
\hline 8 & 38 & $\begin{array}{c}\text { 3/31 (10) E. cloacae complex (1); } \\
\text { E. coli (2) }\end{array}$ & 2/7 (29) E. cloacae complex (3) & Not sampled & $5 / 38(13)$ \\
\hline Total & 228 & 18/161 (11) & $9 / 47(19)$ & $1 / 20(5)$ & 28/228(12) \\
\hline
\end{tabular}

a One animal shed two bacterial species. ${ }^{b}$ Three animals shed two bacterial species each, and one animal shed one bacterial species.

TABLE 3 | Associations and risk factor analysis for ESBL-E/AmpC shedding.

\begin{tabular}{|c|c|c|c|c|c|c|c|c|}
\hline \multirow[t]{2}{*}{ Risk factor/ Sampling site } & \multicolumn{3}{|c|}{ AmpC shedding } & \multicolumn{3}{|c|}{ ESBL shedding } & \multirow[t]{2}{*}{ Overall resistance shedding } & \multirow[t]{2}{*}{ Logistic regression OR } \\
\hline & Gut & Skin/fur/feathers & Total & Gut & Skin/fur/feathers & Total & & \\
\hline Antibiotic treatment ${ }^{a}$ & $0.012^{e}$ & 0.128 & $0.011^{e}$ & 0.069 & 0.457 & $0.038^{e}$ & $0.029^{e}$ & 7.34 \\
\hline Permitted petting policy & $0.002^{\mathrm{e}}$ & 0.6 & $0.015^{\mathrm{e}}$ & $0.012^{\mathrm{e}}$ & 0.58 & 0.057 & $0.021^{e}$ & Not included \\
\hline Petting zoo & 0.186 & 0.191 & $0.017^{e}$ & 0.073 & 0.073 & $0.039^{e}$ & 0.077 & $0.078^{b}$ \\
\hline Animal class ${ }^{c}$ & 0.255 & $0.024^{e}$ & 0.055 & 0.25 & 1 & 0.269 & 0.215 & Not included ${ }^{f}$ \\
\hline Animal diet ${ }^{d}$ & 0.802 & 0.251 & 0.5 & 0.629 & 0.159 & 0.26 & 0.302 & Not included ${ }^{f}$ \\
\hline Animal gender & 0.914 & 0.351 & 1 & 0.641 & 1 & 0.927 & 1 & Not included ${ }^{f}$ \\
\hline
\end{tabular}

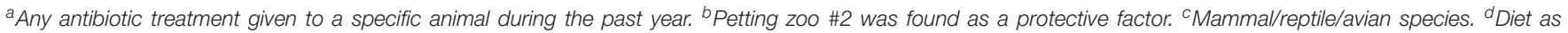

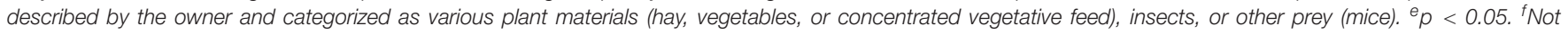
included: excluded from the logistic regression model due to $p>0.2$.

\section{DISCUSSION}

This prospective study investigated the shedding of ESBL/AmpCproducing Enterobacteriaceae in a large and highly diverse sample of petting zoo animal species. To the best of our knowledge, this is the first study that focuses specifically on ESBL/AmpC-E shedding and defines the related risk factors. Of the 228 animals sampled throughout the country, $12 \%$ shed ESBL/AmpC-E. The prevalence of animal shedding varied significantly between different petting zoos, which may be explained by the diverse facilities that were sampled and that represent various animal-visitor interfaces. Because data regarding the prevalence of ESBL/AmpC-E in petting zoos in other countries are unavailable, these data are incomparable with other studies.

Although ESBL/AmpC-E shedding was previously reported in various mammals, reptiles, and avian species (Vittecoq et al., 2016), we screened a highly diverse population of new animal species. We report, for the first time, ESBL/AmpC-E shedding in 13 new host species of mammals, reptiles, and avian species (Figure 1). We found that ESBL/AmpC-E gut shedding was independent of the type of animal species, possibly due to the small number of individual animals sampled within each species. The study included both fecal and surface sampling of diverse animal species, from smooth-skin reptiles to feathered birds or large furred animals, such as sheep or deer. Therefore, ESBL/AmpC-E recovery could be influenced by the type and area of the sampled surface. Supporting this claim is the finding that, in seven animals from four petting zoos, we recovered two different ESBL/AmpC-E strains from the feces and body surface of the same animal, indicating that ESBL/AmpC-E surface shedding may be due to fecal or environmental contamination, rather than gut shedding. In spite of these obstacles, the presence of MDR bacteria on animal surfaces highlights the potential risk of ESBL/AmpC transmission from healthy shedding animals to children and other visitors due to the possible close contact through petting and animal holding.

Importantly, all $35 \mathrm{ESBL} / \mathrm{AmpC}-\mathrm{E}$ isolates recovered from animals belonged to only three different genera - Enterobacter cloacae, E. coli, and C. freundii - with E. cloacae being the most prevalent species, and which was found to be significantly associated with gut shedding. Enterobacter was previously reported to be a commensal bacteria shed by animals in zoos (Ahmed et al., 2007) and a pathogen causing infections in 
animals (Gibson et al., 2010), but it is less frequently reported as a major ESBL/AmpC-producing genus in animals. The main investigated and reported ESBL species in the literature is E. coli. However, in this study we sampled a variety of different species, representing a variety of environmental conditions and interfaces. E. cloacae is ubiquitous in terrestrial and aquatic environments, such as water, sewage, soil, and food (Davin-Regli and Pagès, 2015). We hypothesize that due to the heterogeneous study population, we detected a high prevalence of E. cloacae. Previous reports on zoonotic bacterial outbreaks in petting zoos focused on highly transmissible virulent pathogens, such as E. coli O157:H7 (Stirling et al., 2007; Conrad et al., 2016), Salmonella spp., Campylobacter (Bender and Shulman, 2004), and Shigella (Stirling et al., 2007). Although Enterobacter and Citrobacter are known to be AmpC-producing human pathogens (Santos et al., 2015), they were not described previously as potential zoonotic bacteria. In light of our findings, these genera should be recognized as a possible source for ESBL/AmpC and it is recommended that they be actively monitored in petting zoo facilities.

Bacterial genotyping revealed multiple sequence types, which varied both between and within the same facility. Alarmingly, among the different ESBL-producing E. coli STs recovered, we identified three distinct pathogenic E. coli strains: ETEC ST656 (Oh et al., 2014) and UPEC ST127 (Gibreel et al., 2012), which have both been described as highly virulent, and ST648, which has previously been reported in humans and in domestic and wild animals (Ewers et al., 2014). Each of these three highly virulent $E$. coli STs was recovered from the same mammalian species, housed in the same cage, demonstrating inter-animal clonal transmission that could be explained by animal-to-animal contact or by environmental shedding. These strains were found in two petting zoos (\#7 and \#8) that had a permitted petting policy; thus, transmission may be a relevant risk and could be mediated, e.g., via workers and environmental shedding. In addition, we identified five new E. cloacae STs and one new $C$. freundii ST, which may suggest that these are commensal/environmental strains that may have acquired the ESBL/AmpC resistance via plasmid or gene acquisition. The high diversity of bacterial STs and resistance genes indicates the complex transmission mechanisms and the possible involvement of horizontal transfer of ESBL/AmpC genes and plasmids among petting zoo animals.

We found antibiotic treatment to be a risk factor for ESBL/AmpC-E shedding, similar to previous data on ESBL shedding in both animals (Belas et al., 2014) and humans (Ben-Ami et al., 2009). Assuming that human-animal contact is a risk factor for bacterial transmission in petting zoos, antibiotic-treated animals may constitute a high-risk population for resistant bacterial transmission. In light of these findings, it may be beneficial to consider the interactions between antibiotic-treated animals and visitors. In addition, we found that such animals are treated with a variety of antimicrobials, including third-generation cephalosporins and quinolones. An appropriate guideline for antimicrobial use in petting zoos is currently lacking, and our findings call for the establishment of such a guideline. A recommendation for isolation of antibiotic-treated animals should further studied, since the duration of ESBL/AmpC shedding in animals was not established.

Another important finding was that ESBL/AmpC-E shedding was significantly associated with the petting permission policy. Strong support for this correlation resides in our findings that petting zoo \#2, which had a petting prohibition policy, was found to be a protective factor for $\mathrm{ESBL} / \mathrm{AmpC}-\mathrm{E}$ carriage. We also found ESBL/AmpC-E carriage on the surfaces of animals (skin, fur, or feathers) - an exceptionally important finding in petting zoos, where direct contact is the main interaction between visitors (mostly children) and animals. These findings emphasize the connection between humananimal contact and ESBL/AmpC-E shedding, and they further highlight the importance of implementing strict hygiene and prevention guidelines.

In summary, the data reported in the current study raise new concerns regarding petting zoos as possible sources for ESBL/AmpC-E due to environmental shedding. Considering the valuable contribution of animal-associated activities to physical, social, and psychological aspects of human health (Friedmann et al., 2015), the educational and psychological importance of petting zoos is unequivocal. Therefore, we highly recommend promoting appropriate guidelines and interventions, some of which may include immediate actions and should be further investigated and implemented in the future. Immediate recommendations for petting zoo owners constitute the implementation of hygiene guidelines, including accessible means for hand washing and disinfection, as well as restricted refreshment areas for visitors. Long-term recommendations may comprise improved antibiotic stewardship and the implementation of active surveillance programs. Our findings emphasize the need of additional national and international surveillance studies, which, together, should facilitate the establishment of a standard comprehensive guideline for petting zoo operators and visitors, so as to minimize the associated public health risks.

\section{DATA AVAILABILITY STATEMENT}

The datasets generated for this study can be found in the https:// pubmlst.org/. Accession numbers: ST1151-ST1154 and ST1189.

\section{ETHICS STATEMENT}

The animal study was reviewed and approved by the Internal Ethics Committee of the Koret School of Veterinary Medicine, Israel (Protocol KSVM-VTH/25_2016).

\section{AUTHOR CONTRIBUTIONS}

AS-T and GM collected and analyzed the specimens and petting zoo owners' questioners. AS-T performed the molecular 
analysis. YP performed the bacterial identification. AS-T and WA performed the statistical analysis. AS-T, AS, and SN-V contributed to the conception and design of the study. AS and $\mathrm{SN}-\mathrm{V}$ wrote the manuscript. All authors read and approved the submitted manuscript.

\section{FUNDING}

This study was supported by a research program for $\mathrm{SN}-\mathrm{V}$ funded by the Ariel University grant and a research program for AS funded by The Hebrew University of Jerusalem.

\section{REFERENCES}

Ahmed, A. M., Motoi, Y., Sato, M., Maruyama, A., Watanabe, H., Fukumoto, Y., et al. (2007). Zoo animals as reservoirs of gram-negative bacteria harboring integrons and antimicrobial resistance genes. Appl. Env. Microbiol. 73, 66866690. doi: 10.1128/AEM.01054-1057

Bai, L., Xia, S., Lan, R., Liu, L., Ye, C., Wang, Y., et al. (2012). Isolation and characterization of cytotoxic, aggregative citrobacter freundii. PLoS One 7:e33054. doi: 10.1371/journal.pone.0033054

Barco, L., Belluco, S., Roccato, A., and Ricci, A. (2015). A systematic review of studies on Escherichia coli and Enterobacteriaceae on beef carcasses at the slaughterhouse. Int. J. Food Microbiol. 207, 30-39. doi: 10.1016/j.ijfoodmicro. 2015.04.027

Belas, A., Salazar, A. S., Gama, L. T., Couto, N., and Pomba, C. (2014). Risk factors for faecal colonisation with Escherichia coli producing extended-spectrum and plasmid-mediated AmpC $\beta$-lactamases in dogs. Vet. Rec. 175:202. doi: 10.1136/ vr. 101978

Ben-Ami, R., Rodríguez-Baño, J., Arslan, H., Pitout, J. D. D., Quentin, C., Calbo, E. S., et al. (2009). A multinational survey of risk factors for infection with extended-spectrum $\beta$-lactamase-producing Enterobacteriaceae in nonhospitalized patients. Clin. Infect. Dis. 49, 682-690. doi: 10.1086/604713

Bender, J. B., and Shulman, S. A. (2004). Reports of zoonotic disease outbreaks associated with animal exhibits and availability of recommendations for preventing zoonotic disease transmission from animals to people in such settings. J. Am. Vet. Med. Assoc. 224, 1105-1109. doi: 10.2460/javma.2004.224. 1105

CLSI, (2017). Performance Standards for Antimicrobial Susceptibility Testing. 474 M100 -S27. Wayne, PA: Clinical and Laboratory Standards Institute.

Cole, J. R., Chai, B., Farris, R. J., Wang, Q., Kulam, S. A., McGarrell, D. M., et al. (2005). The ribosomal database project (RDP-II): sequences and tools for high-throughput rRNA analysis. Nucleic Acids Res. 33, D294-D296. doi: 10.1093/nar/gki038

Conrad, C. C., Stanford, K., Narvaez-Bravo, C., Callaway, T., and McAllister, T. (2016). Farm fairs and petting zoos: a review of animal contact as a source of zoonotic enteric disease. Foodborne Pathog. Dis. 14, 59-73. doi: 10.1089/fpd. 2016.2185

Conrad, C. C., Stanford, K., Narvaez-Bravo, C., Neumann, N. F., Munns, K., Tymensen, L., et al. (2018). Zoonotic fecal pathogens and antimicrobial resistance in canadian petting zoos. Microorganisms 6:E70. doi: 10.3390/ microorganisms6030070

Davin-Regli, A., and Pagès, J.-M. (2015). Enterobacter aerogenes and Enterobacter cloacae; versatile bacterial pathogens confronting antibiotic treatment. Front. Microbiol. 6:392. doi: 10.3389/fmicb.2015.00392

Diancourt, L., Passet, V., Verhoef, J., Grimont, P. A. D., and Brisse, S. (2005). Multilocus sequence typing of Klebsiella pneumoniae nosocomial isolates. J. Clin. Microbiol. 43, 4178-4182. doi: 10.1128/JCM.43.8.4178-4182.2005

Erdozain, G., KuKanich, K., Chapman, B., and Powell, D. (2013). Observation of public health risk behaviours, risk communication and hand hygiene at kansas and missouri petting zoos-2010-2011. Zoonoses Public Health 60, 304-310. doi: $10.1111 / \mathrm{j} .1863-2378.2012 .01531 . \mathrm{x}$

\section{ACKNOWLEDGMENTS}

We are grateful to all petting zoo owners, employees, and veterinarians for their collaboration in conducting this study. We are also grateful to Kira Kondratyeva (Ariel University) for her assistance in graphics.

\section{SUPPLEMENTARY MATERIAL}

The Supplementary Material for this article can be found online at: https://www.frontiersin.org/articles/10.3389/fmicb. 2019.02488/full\#supplementary-material

Ewers, C., Bethe, A., Stamm, I., Grobbel, M., Kopp, P. A., Guerra, B., et al. (2014). CTX-M-15-D-ST648 Escherichia coli from companion animals and horses: another pandemic clone combining multiresistance and extraintestinal virulence? J. Antimicrob. Chemother. 69, 1224-1230. doi: 10.1093/jac/dkt516

Friedman, C. R., Torigian, C., Shillam, P. J., Hoffman, R. E., Heltzel, D., Beebe, J. L., et al. (1998). An outbreak of salmonellosis among children attending a reptile exhibit at a zoo. J. Pediatr. 132, 802-807. doi: 10.1016/s0022-3476(98)70307-5

Friedmann, E., Son, H., and Saleem, M. (2015). "Chapter 7 - the animal-human bond: health and wellness," in Handbook on Animal-Assisted Therapy, 4th Edn, ed. A. H. Fine, (San Diego, CA: Academic Press), 73-88.

Gibreel, T. M., Dodgson, A. R., Cheesbrough, J., Fox, A. J., Bolton, F. J., and Upton, M. (2012). Population structure, virulence potential and antibiotic susceptibility of uropathogenic Escherichia coli from northwest England. J. Antimicrob. Chemother. 67, 346-356. doi: 10.1093/jac/dkr451

Gibson, J. S., Cobbold, R. N., Heisig, P., Sidjabat, H. E., Kyaw-Tanner, M. T., and Trott, D. J. (2010). Identification of Qnr and AAC(6')-1b-cr plasmid-mediated fluoroquinolone resistance determinants in multidrug-resistant Enterobacter spp. isolated from extraintestinal infections in companion animals. Vet. Microbiol. 143, 329-336. doi: 10.1016/j.vetmic.2009.11.031

Goode, B., O’Reilly, C., Dunn, J., Fullerton, K., Smith, S., Ghneim, G., et al. (2009). Outbreak of Escherichia coli O157: H7 infections after petting zoo visits, North Carolina state fair, October-November 2004. Arch. Pediatr. Adolesc. Med. 163, 42-48. doi: 10.1001/archpediatrics.2008.525

Heras, J., Domínguez, C., Mata, E., Pascual, V., Lozano, C., Torres, C., et al. (2015). GelJ-a tool for analyzing DNA fingerprint gel images. BMC Bioinformatics 16:270. doi: 10.1186/s12859-015-0703-700

Horton, R. A., Randall, L. P., Snary, E. L., Cockrem, H., Lotz, S., Wearing, H., et al. (2011). Fecal carriage and shedding density of CTX-M extendedspectrum $\beta$-lactamase-producing Escherichia coli in cattle, chickens, and pigs: implications for environmental contamination and food production. Appl. Env. Microbiol. 77, 3715-3719. doi: 10.1128/AEM.02831-2810

Kim, J., Lim, Y.-M., Rheem, I., Lee, Y., Lee, J.-C., Seol, S.-Y., et al. (2005). CTX$\mathrm{M}$ and SHV-12 beta-lactamases are the most common extended-spectrum enzymes in clinical isolates of Escherichia coli and Klebsiella pneumoniae collected from 3 university hospitals within Korea. FEMS Microbiol. Lett. 245, 93-98. doi: 10.1016/j.femsle.2005.02.029

Leonard, E. K., Pearl, D. L., Janecko, N., Finley, R. L., Reid-Smith, R. J., Weese, J. S., et al. (2015). Risk factors for carriage of antimicrobial-resistant Salmonella spp. and Escherichia coli in pet dogs from volunteer households in Ontario, Canada, in 2005 and 2006. Am. J. Vet. Res. 76, 959-968. doi: 10.2460/ajvr.76.11.959

Lin, S.-P., Liu, M.-F., Lin, C.-F., and Shi, Z.-Y. (2012). Phenotypic detection and polymerase chain reaction screening of extended-spectrum $\beta$-lactamases produced by Pseudomonas aeruginosa isolates. J. Microbiol. Immunol. Infect. 45, 200-207. doi: 10.1016/j.jmii.2011.11.015

Miyoshi-Akiyama, T., Hayakawa, K., Ohmagari, N., Shimojima, M., and Kirikae, T. (2013). Multilocus sequence typing (MLST) for characterization of Enterobacter cloacae. PLoS One 8:e66358. doi: 10.1371/journal.pone.0066358

Murk, J.-L., Heddema, E. R., Hess, D. L., Bogaards, J. A., Vandenbroucke-Grauls, C. M., and Debets-Ossenkopp, Y. J. (2009). Enrichment broth improved detection of extended-spectrum-beta-lactamase-producing bacteria in throat 
and rectal surveillance cultures of samples from patients in intensive care units. J. Clin. Microbiol. 47, 1885-1887. doi: 10.1128/JCM.01406-1408

Oh, K.-H., Kim, D. W., Jung, S.-M., and Cho, S.-H. (2014). Molecular characterization of Enterotoxigenic Escherichia coli strains isolated from diarrheal patients in Korea during 2003-2011. PLoS One 9:e96896. doi: 10.1371/ journal.pone.0096896

Santos, G. S. D., Solidônio, E. G., Costa, M. V. S., Melo, R. A., Souza, I. A., de Silva, G. R., et al. (2015). Study of the Enterobacteriaceae Group CESP (Citrobacter, Enterobacter, Serratia, Providencia, Morganella and Hafnia): A Review. Available at: https://www.semanticscholar.org/paper/Study-ofthe-Enterobacteriaceae-Group-CESP-and-A-Santos-Solid\%C3\%B4nio/ 143ee9f2c91bcb7c645f42bc61464365b440c0f3 (accessed August 21, 2018).

Schwaber, M. J., Navon-Venezia, S., Kaye, K. S., Ben-Ami, R., Schwartz, D., and Carmeli, Y. (2006). Clinical and economic impact of bacteremia with extendedspectrum-beta-lactamase-producing Enterobacteriaceae. Antimicrob. Agents Chemother. 50, 1257-1262. doi: 10.1128/AAC.50.4.1257-1262. 2006

Steinmuller, N., Demma, L., Bender, J. B., Eidson, M., and Angulo, F. J. (2006). Outbreaks of enteric disease associated with animal contact: not just a foodborne problem anymore. Clin. Infect. Dis. Off. Publ. Infect. Dis. Soc. Am. 43, 1596-1602. doi: 10.1086/509576

Stirling, J., Griffith, M., Dooley, J. S. G., Goldsmith, C. E., Loughrey, A., Lowery, C. J., et al. (2007). Zoonoses associated with petting farms and open zoos. Vector Borne Zoonotic Dis. 8, 85-92. doi: 10.1089/vbz.2006.0639

Tofteland, S., Haldorsen, B., Dahl, K. H., Simonsen, G. S., Steinbakk, M., Walsh, T. R., et al. (2007). Effects of phenotype and genotype on methods for detection of extended-spectrum-beta-lactamase-producing clinical isolates of Escherichia coli and Klebsiella pneumoniae in Norway. J. Clin. Microbiol. 45, 199-205. doi: 10.1128/JCM.01319-1316

Versalovic, J., Koeuth, T., and Lupski, J. R. (1991). Distribution of repetitive DNA sequences in Eubacteria and application to fingerprinting of bacterial genomes. Nucleic Acids Res. 19, 6823-6831. doi: 10.1093/nar/19.24.6823

Vittecoq, M., Godreuil, S., Prugnolle, F., Durand, P., Brazier, L., Renaud, N., et al. (2016). Antimicrobial resistance in wildlife. J. Appl. Ecol. 53, 519-529. doi: 10.1111/1365-2664.12596

Weese, J. S., McCarthy, L., Mossop, M., Martin, H., and Lefebvre, S. (2007). Observation of practices at petting zoos and the potential impact on zoonotic disease transmission. Clin. Infect. Dis. Off. Publ. Infect. Dis. Soc. Am. 45, 10-15. doi: $10.1086 / 518572$

Woodford, N., Fagan, E. J., and Ellington, M. J. (2006). Multiplex PCR for rapid detection of genes encoding CTX-M extended-spectrum (beta)-Lactamases. J. Antimicrob. Chemother. 57, 154-155. doi: 10.1093/jac/dki412

Conflict of Interest: The authors declare that the research was conducted in the absence of any commercial or financial relationships that could be construed as a potential conflict of interest.

Copyright (๔) 2019 Shnaiderman-Torban, Steinman, Meidan, Paitan, Abu Ahmad and Navon-Venezia. This is an open-access article distributed under the terms of the Creative Commons Attribution License (CC BY). The use, distribution or reproduction in other forums is permitted, provided the original author(s) and the copyright owner(s) are credited and that the original publication in this journal is cited, in accordance with accepted academic practice. No use, distribution or reproduction is permitted which does not comply with these terms. 